\title{
Targeted photocoagulation of peripheral ischemia to treat rebound edema
}

\author{
This article was published in the following Dove Press journal: \\ Clinical Ophthalmology \\ 13 February 2015 \\ Number of times this article has been viewed
}

\author{
Michael A Singer' \\ Colin S Tan ${ }^{2}$ \\ Krishna R Surapaneni ${ }^{3}$ \\ Srinivas R Sadda ${ }^{4}$ \\ IMedical Center Ophthalmology \\ Associates, San Antonio, TX, USA; \\ ${ }^{2}$ National Healthcare Group Eye \\ Institute, Tan Tock Seng Hospital, \\ Singapore; ${ }^{3}$ University of Texas Health \\ Science Center at San Antonio, San \\ Antonio, TX, USA; ${ }^{4}$ Doheny Eye \\ Institute, Los Angeles, CA, USA
}

Introduction: Peripheral retinal ischemia not detectable by conventional fluorescein angiography has been proposed to be a driving force for rebound edema in retinal vein occlusions. In this report, we examine the treatment of peripheral retinal ischemia with targeted retinal photocoagulation (TRP) to manage a patient's rebound edema.

Methods: To assess the extent of peripheral nonperfusion, an Optos 200Tx device was used. To target the treatment to peripheral ischemia areas, a Navilas Panretinal Laser was used.

Results: A 64-year-old male with a central retinal vein occlusion and a visual acuity 20/300, and central macular thickness $318 \mu \mathrm{m}$ presented with rubeosis. Angiography revealed extensive peripheral nonperfusion. Despite TRP to areas of irreversible ischemia, after 2 months, he continued show rubeosis and rebound edema. Additional TRP laser was repeatedly added more posteriorly to areas of reversible nonperfusion, resulting in eventual resolution of rubeosis and edema.

Conclusion: In this study, we demonstrate the use of widefield imaging with targeted photocoagulation of peripheral ischemia to treat rebound edema, while preserving most peripheral vision. In order to treat rebound edema, extensive TRP, across reversible and nonreversible areas of ischemia, had to be performed - not just in areas of nonreversible peripheral ischemia. These areas need to be mapped during episodes of rebound edema, when ischemia is at its maximum. In this way, by doing the most TRP possible, the cycle of rebound edema can be broken.

Keywords: macular edema, retinal vein occlusion

\section{Background and objective}

Retinal vein occlusions (RVOs) are the second most common type of retinal vascular disorders, affecting an estimated 180,000 eyes per year in the United States. ${ }^{1,2}$ Macular edema is a frequent cause of vision loss following RVO and can have a variable clinical course, which may be challenging to manage. ${ }^{3,4}$ Current treatment options include laser photocoagulation, intravitreal corticosteroids, and intravitreal anti-vascular endothelial growth factor (VEGF) antagonists. A challenge has been that premature discontinuation of therapy may result in rebound edema that is often more severe than the initial presentation. ${ }^{5}$ Peripheral ischemia and levels of VEGF have been proposed to be a driving force for rebound edema. ${ }^{6,7}$

Eyes with central RVOs have been classified as "ischemic" versus "nonischemic" or "perfused" versus "nonperfused". ${ }^{8-10}$ The Central Vein Occlusion Study examined the "nonperfused" area within a limited region of the posterior pole of the eye in patients with central RVOs and determined that that the area of nonperfusion was a risk factor for development of neovascularization of the iris and neovascular glaucoma. ${ }^{11}$ The use of anti-VEGF therapy has since decreased the risk of neovascular complications. ${ }^{10}$ However, nonperfusion is believed to be a contributing factor to the need for a higher frequency of anti-VEGF injections. Analysis of fluorescein angiograms
Correspondence: Michael Singer 9157 Huebner Road, San Antonio, TX 78240, USA

Tel +l 2106972020

Fax + I 2105587679

Email msingerII@mac.com 
with a conventional fundus camera has shown that despite there being no observable areas of nonperfusion within the field of view, some patients require frequent and continuous anti-VEGF therapy, suggesting that there may be areas of nonperfusion that remain undetected by conventional imaging. ${ }^{12}$ Studies have shown that there are areas of peripheral retinal ischemia present despite absence of non perfusion on a conventional fluorescein angiogram (FA). These areas of non perfusion can be demonstrated using wide field angiography, which can provide almost a 200 degree view of the posterior pole. ${ }^{13,14}$

Treatment of these areas of peripheral nonperfusion with targeted retinal photocoagulation (TRP) has been suggested as a potentially useful approach in patients with retinal vascular disease. However thus far, there have been mixed results with targeted photocoagulation to areas of nonperfusion. ${ }^{12}$ We previously described that there is considerable variability in the area of peripheral retinal nonperfusion, and this is dynamically related to the severity of edema. ${ }^{15}$ Furthermore in previous studies, the anti-ischemic effects of the last anti-VEGF injection given prior to TRP treatment may have reduced the apparent area of peripheral nonperfusion. Hence, we performed this case study to test the role of maximizing treatment targeted to these reversible areas of ischemia left when the anti-VEGF injection had worn off. In this report, we describe the use of automated targeted TRP of peripheral nonperfusion, guided by widefield angiography, to manage rebound macular edema in a patient refractory to anti-VEGF therapy alone.

\section{Materials and methods}

The patient in this report was also part of a previously described investigator-sponsored study. ${ }^{15}$ The patient was seen and treated at Medical Center Ophthalmology Associates, San Antonio, TX, and the treatment was approved by its Institutional Review Board. Written informed consent was obtained. To image peripheral ischemia, an Optos 200Tx device (Optos, Dunfermline, Scotland, UK) was used at all visits. The Optos 200Tx performs ultra-widefield angiography with 200-degree pole-to-periphery views, compared with conventional FA, which permit 30- to 50-degree posterior pole views. To target treatment to areas of peripheral ischemia, a Navilas Panretinal Laser (OD-OS GmbH, Teltow, Germany) was used, which enabled navigated TRP to the periphery, compared with conventional laser, which has limited planning and treatment documentation. Masked investigators at the Doheny Image Reading Center were enlisted to grade the images and measure the areas of ischemia, using previously described validated planimetric grading software (GRADOR). In accordance with previous studies, the extent of nonperfusion was reported as an ischemic index, which defined the area of ischemia as a percentage of the total visible retina.

\section{Case study and results}

The patient was a 64-year-old male with a central RVO who presented with a visual acuity of 20/200, and central macular thickness of greater than $318 \mu \mathrm{m}$ and volume of $14 \mathrm{~mm}^{3}$. He was also noted to have rubeosis but declined recommended TRP therapy for fear of further loss of peripheral vision. As a result, he elected to have anti-VEGF injections. Optos widefield FA demonstrated a relationship between his optical coherence tomography (OCT) volume and ischemic index, which was $50.6 \%$ on initial presentation (Figure 1). When treated with anti-VEGF agents, his OCT volume decreased to less than $9 \mathrm{~mm}^{3}$, his perfusion improved, and his ischemic index decreased to below $50 \%$ after each treatment. When his antiVEGF therapy wore off, the amount of ischemia increased, his OCT volume increased to over $9 \mathrm{~mm}^{3}$, and his ischemic index increased to over $50 \%$. This phenomenon of a region of apparent reversible nonperfusion following anti-VEGF therapy was a consistent finding, and the cyclical process continued for approximately 1 year. After nine injections and monthly follow up, the patient expressed interest in exploring options to decrease the number of injections, while still preserving as much of his peripheral vision as possible. He elected to have navigated TRP 2 weeks after his anti-VEGF injection. The goal of this treatment strategy was to stop the recurring cycle of rebound edema, while preserving as much peripheral

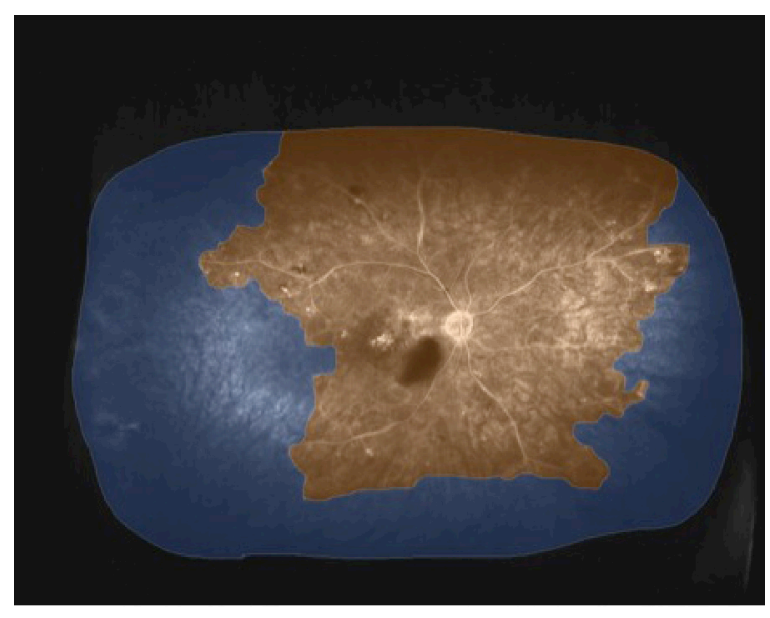

Figure I Widefield FA prior to treatment.

Notes: Areas of nonperfusion are shaded blue, while areas of perfusion are orange. The ischemic index is $50.6 \%$.

Abbreviation: FA, fluorescein angiography. 


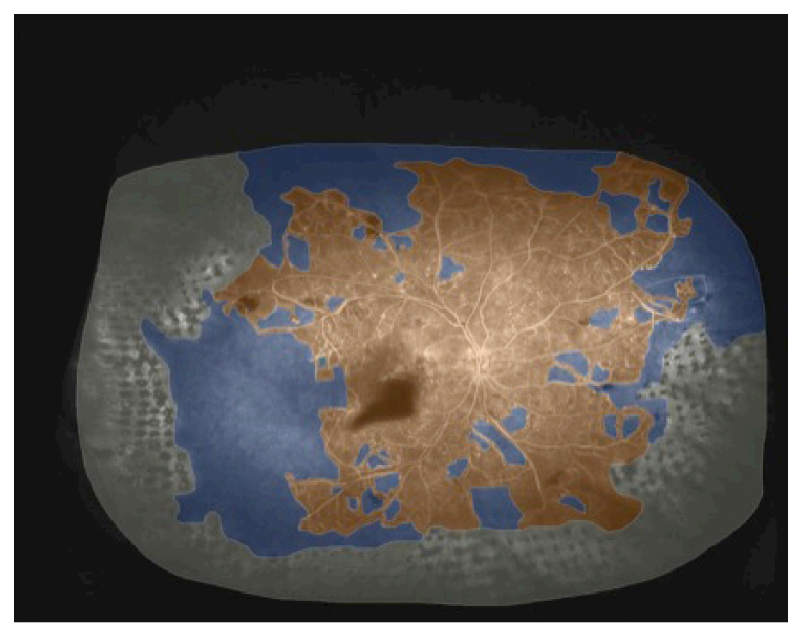

Figure 2 Widefield FA after initial targeted retinal photocoagulation.

Notes: Areas that have been lasered are shown in grey. The ischemic index is $40.9 \%$. Abbreviation: FA, fluorescein angiography.

vision as possible. The rationale for this combination approach was that anti-VEGF therapy would reveal areas of reversible ischemia, which might represent areas of "saveable" retina that could potentially be spared laser therapy. Thus, by only applying laser to areas of nonreversible ischemia, the cycle of rebound edema would hopefully be stopped, with the least amount of peripheral visual field loss.

However 1 month after this guided peripheral TRP, the patient's vision was still 20/200 and his retinal volume had increased to $15.0 \mathrm{~mm}^{3}$, with recurrent rubeosis and an ischemic index of $40.9 \%$ (Figure 2). As a result, additional TRP was applied over multiple sessions over the next several months, extending more posteriorly into the areas of reversible ischemia (Figure 3 ). These additional treatments

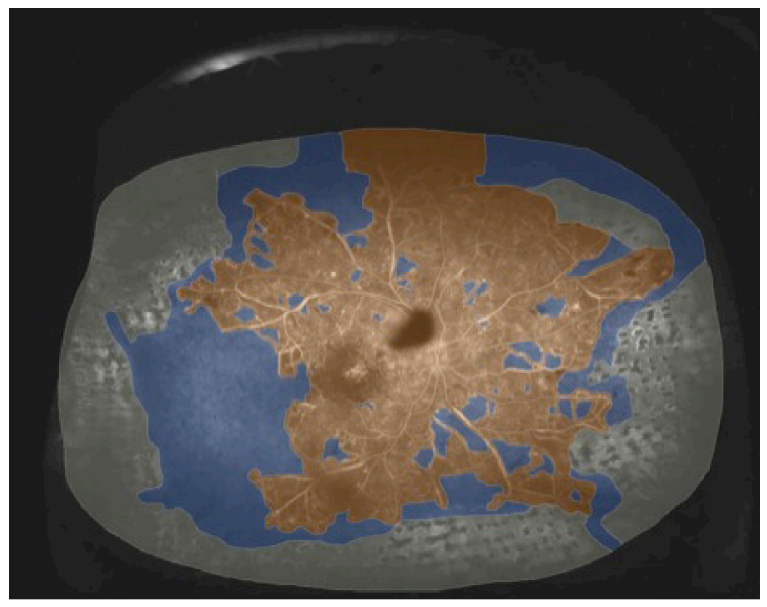

Figure 3 Widefield FA 3 months later, following additional targeted retinal photocoagulation.

Note: The ischemic index is $38.7 \%$.

Abbreviation: FA, fluorescein angiography. were done in a staged fashion in the hopes of treating the minimal amount of retina possible to achieve the desired goal of absence of recurrent rubeosis and edema. Ultimately, recurrent rubeosis and edema were prevented only when TRP was given to most of the area of reversible ischemia (Figure 4) in addition to the previously lasered nonreversible ischemic zones. To ensure that the entire zone (reversible and nonreversible) of nonperfusion was included in the treatment plan, Optos widefield FA was obtained 2 months after the last anti-VEGF injection, when the ischemic area, ischemic index and OCT volume were at their highest. Following completion of this staged targeted retinal photocoagulation, the residual ischemic index was reduced to $14.2 \%$ of the nonlasered retinal area. To date, the patient has been followed for over 1 year and has not needed additional injections.

\section{Discussion}

Rebound edema was first described by Matsumoto et al after an observation that patients who were treated for RVO with anti-VEGF injections developed increased edema on OCT after anti-VEGF therapy was discontinued. The authors postulated that rebound edema was due to untreated peripheral ischemia, which caused VEGF production, leading to ischemia. ${ }^{5}$ It is known that TRP therapy reduces VEGF production in the retina by reducing tissue oxygen demand, thereby reducing the ischemic drive. ${ }^{16}$ It has been theorized that this effect could potentially break the cycle of rebound edema. However studies have not demonstrated this to be the case. ${ }^{12}$ We have previously shown ${ }^{15}$ that the use of anti-VEGF agents actually causes a decrease in the amount of ischemia seen on Optos widefield angiography. In a prospective cohort

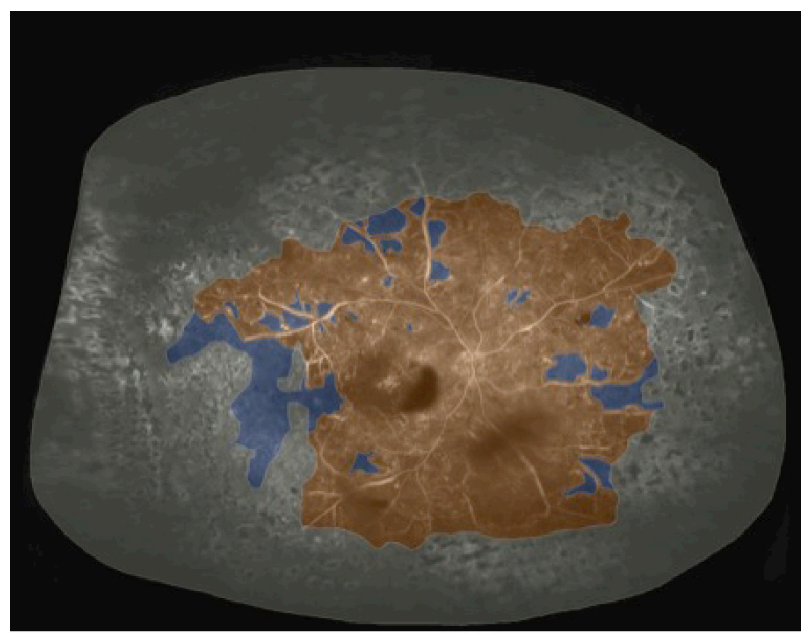

Figure 4 Widefield FA after completion of targeted retinal photocoagulation.

Note: The ischemic index is $14.2 \%$.

Abbreviation: FA, fluorescein angiography. 


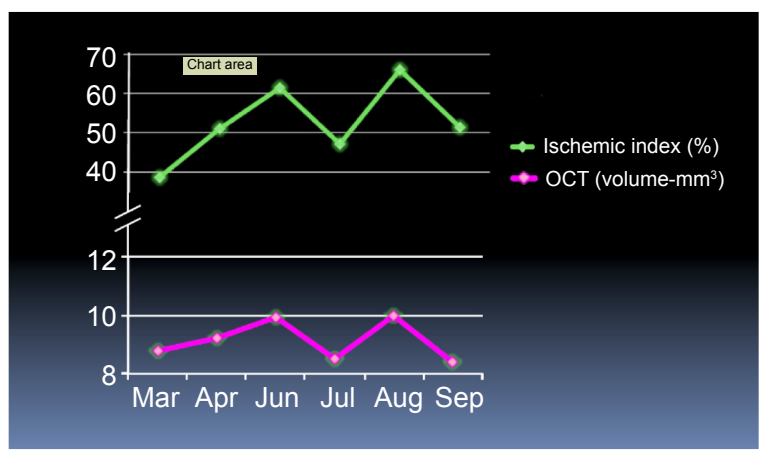

Figure 5 Variation of OCT thickness with the extent of ischemia. Abbreviation: OCT, optical coherence tomography.

study, mean ischemic index was reduced from $14.8 \%$ on initial presentation to $10.3 \%$ following treatment with antiVEGF agents. ${ }^{15}$ In the present study, we also showed that the level of nonperfusion correlates with the severity of macular edema (Figure 5).

In our particular patient, numerous attempts at TRP were performed to eliminate the source of edema, each of them using widefield angiography as a guide. Only when the amount of TRP nearly equaled the maximum amount of ischemia, which was present when the effects of anti-VEGF wore off, were we able to eliminate the cycle of rebound edema and rubeosis. It is, however, possible that rebound edema may be unrelated to the TRP treatments; the macular edema may have either resolved over time, or it may be that the patient's peripheral retina was producing VEGF and may have finally autoinfarcted.

The fact that we were able to break the rebound edema cycle while other groups were not may be explained by the fact that in other studies, the anti-ischemic effects of anti-VEGF agents may have still been on board, causing the Optos FA to appear less ischemic than it actually was. As a result, the amount of TRP used by others may have been insufficient and therefore, they undertreated the true area of ischemia.

In this study, we demonstrate the integrated use of Optos 200Tx widefield imaging with the Navilas Panretinal laser to treat peripheral retinal ischemia and subsequently, to break the cycle of rebound edema, while preserving most peripheral vision. This case study shows that in order to prevent rebound edema, both the nonreversible and reversible areas of peripheral nonperfusion need to be treated. To define these areas for targeted treatment, the timing of widefield angiography with respect to anti-VEGF therapy must be carefully considered. In particular, it is crucial to map the areas of nonperfusion when the edema (and presumably the
VEGF in the eye) is at its maximum. By using this strategy for TRP, we suggest that the cycle of rebound edema may be potentially broken. Subsequent prospective studies will be crucial for validating our hypothesis.

\section{Disclosure}

M Singer has been a consultant for Allergan and Valeant; and has received research support from Alcon and Allergan. C Tan received research support from a National Healthcare Group Clinician Scientist Career Scheme Grant (grant number CSCS 12-005) and conference support from Bayer, Heidelberg Engineering, and Novartis. S Sadda has been a consultant for Allegan, Genentech, Roche, Regeneron, Alcon, Bausch\&Lomb, Optos, and Carl Zeiss Meditec, and has received research support from Allegan, Genentech, Optos, and Carl Zeiss Meditec. The authors report no other conflicts of interest in this work.

\section{References}

1. Klein R, Moss SE, Meuer SM, Klein BE. The 15-year cumulative incidence of retinal vein occlusion: the Beaver Dam Eye Study. Arch Ophthalmol. 2008;126(4):513-518.

2. US Census Bureau. Annual Estimates of the population by sex and fiveyear age groups for the United States: April 1, 2000 to July 1, 2007. Suitland, MD: US Census Bureau; 2013.

3. Rehak J, Rehak M. Branch retinal vein occlusion: pathogenesis, visual prognosis, and treatment modalities. Curr Eye Res. 2008;33(2): 111-131.

4. Mohamed Q, McIntosh RL, Saw SM, Wong TY. Interventions for central retinal vein occlusion: an evidence-based systematic review. Ophthalmology. 2007;114(3):507-19, 524.

5. Matsumoto Y, FreundKB, PeirettiE, Cooney MJ, Ferrara DC, YannuzziLA. Rebound macular edema following bevacizumab (Avastin) therapy for retinal venous occlusive disease. Retina. 2007;27(4):426-431.

6. Koss MJ, Pfister M, Rothweiler F, et al. Comparison of cytokine levels from undiluted vitreous of untreated patients with retinal vein occlusion. Acta Ophthalmol. 2012;90(2):e98-e103.

7. Boyd SR, Zachary I, Chakravarthy U, et al. Correlation of increased vascular endothelial growth factor with neovascularization and permeability in ischemic central vein occlusion. Arch Ophthalmol. 2002; 120(12):1644-1650.

8. Hayreh SS. Classification of central retinal vein occlusion. Ophthalmology. 1983;90(5):458-474.

9. Hayreh SS, Klugman MR, Beri M, Kimura AE, Podhajsky P. Differentiation of ischemic from non-ischemic central retinal vein occlusion during the early acute phase. Graefes Arch Clin Exp Ophthalmol. 1990; 228(3):201-217.

10. Risard SM, Pieramici DJ, Rabena MD, et al. Intravitreal ranibizumab for macular edema secondary to central retinal vein occlusion. Retina. 2011;31(6):1060-1067.

11. A randomized clinical trial of early panretinal photocoagulation for ischemic central vein occlusion. The Central Vein Occlusion Study Group N report. Ophthalmology. 1995;102(10):1434-1444.

12. Spaide RF. Prospective study of peripheral panretinal photocoagulation of areas of nonperfusion in central retinal vein occlusion. Retina. 2013; 33(1):56-62.

13. Prasad PS, Oliver SC, Coffee RE, Hubschman JP, Schwartz SD. Ultra wide-field angiographic characteristics of branch retinal and hemicentral retinal vein occlusion. Ophthalmology. 2010;117(4):780-784. 
14. Tsui I, Kaines A, Havunjian MA, et al. Ischemic index and neovascularization in central retinal vein occlusion. Retina. 2011;31(1): 105-110.

15. Singer M, Tan CS, Bell D, Sadda SR. Area of peripheral retinal nonperfusion and treatment response in branch and central retinal vein occlusion. Retina. 2014;34(9):1736-1742.
16. one.aao.org [homepage on the Internet]. Proliferative and non proliferative diabetic retinopathy. ONE Network; 2013 [updated October 22, 2013; cited September 3, 2014]. Available from: http://one.aao.org/ munnerlyn-laser-surgery-center/laser-treatment-of-proliferativenonproliferative-. Accessed December 9, 2014.

\section{Publish your work in this journal}

Clinical Ophthalmology is an international, peer-reviewed journal covering all subspecialties within ophthalmology. Key topics include: Optometry; Visual science; Pharmacology and drug therapy in eye diseases; Basic Sciences; Primary and Secondary eye care; Patient Safety and Quality of Care Improvements. This journal is indexed on

Submit your manuscript here: http://www.dovepress.com/clinical-ophthalmology-journal

\section{Dovepress}

PubMed Central and CAS, and is the official journal of The Society of Clinical Ophthalmology (SCO). The manuscript management system is completely online and includes a very quick and fair peer-review system, which is all easy to use. Visit http://www.dovepress.com/ testimonials.php to read real quotes from published authors. 